\title{
Altered spontaneous brain activity in patients with strabismic amblyopia: A resting-state fMRI study using regional homogeneity analysis
}

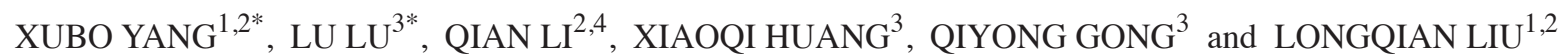 \\ Departments of ${ }^{1}$ Ophthalmology and ${ }^{2}$ Optometry and Visual Science; \\ ${ }^{3}$ Huaxi MR Research Center (HMRRC), Department of Radiology, West China Hospital of Sichuan University, \\ Chengdu, Sichuan 610041; ${ }^{4}$ Department of Ophthalmology, Henan Provincial People's Hospital, \\ Zhengzhou, Henan 450003, P.R. China
}

Received October 25, 2018; Accepted August 21, 2019

DOI: $10.3892 /$ etm.2019.8038

\begin{abstract}
The present study aimed to compare the regional homogeneity (ReHo) of resting-state brain function between subjects with strabismic amblyopia and normal controls. A total of 12 adult patients with strabismic amblyopia and 34 age- and gender-matched normal controls were enrolled in the present study. All patients were subjected to resting-state magnetic resonance imaging using a Siemens Trio 3.0T magnetic resonance scanner. ReHo values of the whole brain were calculated and standardized. An independent-samples t-test was used to analyze the differences in ReHo values between the two groups. Patients with strabismic amblyopia exhibited reduced ReHo values in certain parts of the occipital lobe, including the lingual gyrus, cuneus and superior occipital gyrus. Increased ReHo values were observed in the precuneus and certain parts of the prefrontal cortex of patients with strabismic amblyopia, including the superior frontal gyrus and middle frontal gyrus. The ReHo index of the precuneus was negatively correlated with age. However, there was no correlation between the ReHo values and the visual acuity of patients with strabismic amblyopia. ROC curve analysis demonstrated that the greatest area under curve (AUC) value was in the medial prefrontal cortex $(\mathrm{AUC}=0.864)$. The results of the present study suggested that visual information processing may be impaired in visual areas V1 and V2. Furthermore, adult patients with strabismic amblyopia exhibited brain
\end{abstract}

Correspondence to: Professor Longqian Liu, Department of Ophthalmology, West China Hospital of Sichuan University, 37 Guoxue Xiang, Chengdu, Sichuan 610041, P.R. China

E-mail: b.q15651@hotmail.com

*Contributed equally

Key words: strabismic amblyopia, regional homogeneity, functional magnetic resonance imaging, resting state plasticity that compensated for visuomotor coordination and visuospatial imagery deficits.

\section{Introduction}

Amblyopia is a vision development disorder caused by abnormal visual experience during the sensitive period, affecting $3 \%$ of the world's population. The neural mechanism underlying amblyopia remains to be determined; however, a number of studies have aimed to identify the localization of the neurological deficit (1-17).

Previous studies reported reduced visual cortex gray matter volume (1-3), as well as poor white-matter development among patients with amblyopia $(3,4)$. These morphological alterations of the visual cortex may be associated with the visual deficits in amblyopia.

Functional magnetic resonance imaging (fMRI) is a non-invasive method frequently used to determine the functional alterations of cerebral areas. The majority of previous studies on amblyopia used task-based fMRI. Certain studies indicated that this disease may originate from the striate cortex $(5,6)$; however, neurological deficits were also identified in the lateral geniculate nucleus $(7,8)$ and extrastriate cortex $(9,10)$.

Task-based fMRI is a modified fMRI analysis based on stimulation or task performance. Lack of patient cooperation and challenges associated with task standardization limit the clinical applicability of task-based fMRI. Therefore, this type of fMRI is not extensively used in the diagnosis of human diseases and associated research. The human brain constitutes $\sim 2 \%$ of the human body weight; however, it consumes $\sim 20 \%$ of the total energy expenditure. The greatest amount of energy is required for information transfer between neurons. Energy consumption during cognitive task performance increases by $5 \%$ compared with resting-state energy expenditure (11). Therefore, task-based fMRI investigates part of the brain activity, whereas a comprehensive brain analysis may be performed using resting-state fMRI.

A number of studies investigated the underlying neurological mechanisms of amblyopia using resting-state fMRI. Lin et al (12) investigated alterations in resting-state local 
spontaneous brain activity among patients with anisometropic amblyopia using regional homogeneity (ReHo) values. The results of this study indicated decreased spontaneous brain activity represented by low ReHo values of certain brain regions, including the precuneus, medial prefrontal cortex and cerebellum (12). However, compensatory plasticity was detected in other areas of the brain, including the bilateral conjunction area of the post-central and pre-central gyri, paracentral lobule and superior temporal gyrus (12). Ding et al (13) further investigated the functional connectivity of the primary visual area of patients with amblyopia using resting-state fMRI. The results demonstrated altered functional connectivity of the cerebellum, inferior parietal lobule and primary visual cortex among individuals with amblyopia (13). Wang et al (14) used functional-connectivity density mapping and an ultrafast data-driven method based on resting-state fMRI to investigate alterations in cortical functional connectivity among patients with anisometropic amblyopia. This study identified impaired short-range functional connections in the visual pathways of the brain and in the frontal cortex, and altered long-range functional connections among the visual regions, posterior parietal and frontal cortices of patients with amblyopia (14). A previous study by our group used resting-state fMRI combined with amplitude of low-frequency fluctuation (ALFF) analysis to investigate the intrinsic brain activity patterns of patients with amblyopia and the results indicated an impaired function of certain brain regions and increased ALFF of other brain areas (15). Similar data were reported in Liang et al (16). Recently, a novel voxel-wise image analysis method, voxel-mirrored homotopic connectivity (VMHC), was used to assess the alterations in resting-state functional connectivity in patients with amblyopia (17). The results revealed similarities and differences in interhemispheric functional connectivity between patients with anisometropic and strabismic amblyopia (17).

Based on the hypothesis that brain activity occurs in clusters, as opposed to a single voxel, Zang et al (18) proposed that the ReHo index may be used for the analysis of brain activity patterns during the resting state. The ReHo index evaluates the temporal homogeneity between the time series of each voxel and its nearest neighbors, to reflect the regional activity of the whole brain. The ReHo index indirectly reflects the homogeneity of blood oxygen level-dependent signals, and alterations of ReHo values may suggest abnormal activity of neurons.

The ReHo index has been used to evaluate diverse neurological and psychiatric diseases, including schizophrenia (19), attention deficit hyperactivity disorder (20), Parkinson's disease (21) and Alzheimer's disease (22). Furthermore, this index has been used in the field of ophthalmology to investigate blindness (23), glaucoma (24), strabismus (25) and anisometropic amblyopia (12). The above studies used the ReHo index to obtain novel neuropathological data.

The ReHo values of spontaneous brain activity of patients with strabismic amblyopia remain elusive. Furthermore, strabismic and anisometropic amblyopia are characterized by different early abnormal visual experiences. A previous study revealed differences between these conditions using resting-state fMRI combined with VMHC analysis (17). Therefore, the spontaneous activity pattern of patients with strabismic amblyopia may differ from that of patients with anisometropic amblyopia. In the present study, the ReHo index was used to analyze the brain activity of patients with strabismic amblyopia.

\section{Patients and methods}

Patients. Patients with strabismic amblyopia were recruited between June 2015 and November 2017 from the Department of Ophthalmology of West China Hospital of Sichuan University (Chengdu, China) and diagnosed using the Expert Consensus on Amblyopia Diagnosis guidelines (26). The normal control group included healthy student volunteers recruited from Sichuan University between January 2016 and December 2017.

All participants were subjected to eye examination, including a visual acuity test, ocular tonometry, slit-lamp examination, dilated fundus examination, cover test and ocular movement examination. Furthermore, the near point of convergence, stereoacuity and refraction were determined for each patient. A total of 12 patients with strabismic amblyopia and 34 normal controls were enrolled in the present study. A total of 1 patient with strabismic amblyopia and 7 healthy volunteers were excluded due to excessive head motion. The patients included in the present study were right-handed and aged 18-35 years. Patients with strabismic amblyopia exhibited an ocular deviation of $\geq 15 \Delta$ and normal controls exhibited naked eye or corrected visual acuity of $\geq 1.0$ in the bilateral eyes. The exclusion criteria were as follows: i) A history of other ocular diseases; ii) strabismus identified among control patients; iii) a history of neurological diseases; iv) claustrophobia; and v) inability to cooperate.

The protocol of the present study was approved by the Ethics Committee of West China Hospital of Sichuan University (Chengdu, China) and complied with the principles of the Declaration of Helsinki. Written informed consent was voluntarily provided by all participants after the purpose and methods of the present study were explained verbally and in writing.

MRI data acquisition. All MRI data analyses were performed at the MR Research Center of West China Hospital using the Magnetom Trio 3.0T MRI system (Siemens Healthineers) equipped with a standard eight-channel quadrature head coil. The imaging area covered the entire brain. All participants were informed of the contraindications of the MRI examination and other associated matters of importance. The MRI examination followed the standardized scanning procedures (27).

MRI scout images and three-dimensional structural T1-weighted images were initially obtained, followed by resting-state fMRI scanning. Structural T1-weighted images were obtained using a magnetization-prepared rapid gradient-echo sequence with the following parameters: Echo time (TE), $2.62 \mathrm{msec}$; repetition time (TR), 2,250 msec; field of view (FOV), 25.6x25.6 cm; flip angle (FA), $9^{\circ}$; matrix, $256 \times 256$. Resting-state functional images were obtained using an echo-planar imaging sequence with the following parameters: TE, $35 \mathrm{msec}$; TR, 2,000 msec; FOV, 20.8×20.8 cm; FA, $68^{\circ}$; matrix, $64 \times 64$.

Data pre-processing. Prior to data pre-processing, data in DICOM format were converted to the NIFTI format 
using MRIcron software v. 12/2012 (www.cabiatl. $\mathrm{com} / \mathrm{mricro} / \mathrm{mricron} / \mathrm{dcm} 2 \mathrm{nii}$.html). Pre-processing was performed using the Data Processing Assistant for Resting State fMRI software 2.2 (rfmri.org/DPARSF) based on Statistical Parametric Mapping 8 (www.fil.ion.ucl.ac.uk/spm).

Due to initial transient signal fluctuations and adaptation to the scanning process, the data obtained from the first 10 volumes of each functional time series were discarded and the remaining 210 volumes were processed with slice timing and realignment for head motion correction. Participants with head motion $\leq 2.0 \mathrm{~mm}$ or $\leq 2.0^{\circ}$ rotation in any axis were included. One patient with strabismic amblyopia and seven healthy volunteers were excluded due to excessive head motion.

All realigned data were spatially normalized according to the standard Montreal Neurological Institute (MNI) coordinates and resampled to $3 \times 3 \times 3 \mathrm{~mm}^{3}$ voxels. To reduce the effects of linear tendency due to the long duration of the scan, linear drift was removed from the data. Furthermore, the regressed images were smoothed with an 8 -mm full width at half maximum to remove spatial noise. Finally, a temporal bandpass filter (0.01-0.08 Hz) was used to minimize the effects of low-frequency drift and high-frequency noise.

ReHo index measurement. The ReHo indices of 12 patients with strabismic amblyopia and 34 normal controls were calculated. When calculating the ReHo index, it was assumed that each voxel has a high temporal correlation with its neighboring voxels in the same cerebral region. Kendall's coefficient of concordance (KCC) was used to describe the similarity of time series in a voxel with its 26 neighboring voxels. KCC values range from 0 to 1 , and an increase in the $\mathrm{KCC}$ value indicates an increase in regional homogeneity (18). The whole-brain map of regional brain activity was obtained following calculation of the ReHo index of each voxel. To minimize the influence of individual variability, the ReHo value for each voxel was divided by the mean ReHo of the whole brain for normalization. Normalized ReHo values indicated regional brain activity across the entire brain.

Statistical analysis. Demographic data were compared between patients with strabismic amblyopia and normal controls using SPSS software (version 18.0; SPSS, Inc.). An independent-samples t-test was used for the analysis of numerical variables and a $\chi^{2}$ test was used for categorical variables.

Independent-samples t-tests were performed using Resting State fMRI Data Analysis Toolkit software (version 1.8; restfmri.net/forum/REST_V1.8) to determine the differences in the normalized whole-brain ReHo values between patients with strabismic amblyopia and healthy controls. Initially, $\mathrm{P}<0.01$ was considered to indicate a statistically significant difference. Subsequently, AlphaSim multiple-comparison correction was used and the P-value cut-off was adjusted to 0.05 with a cluster size of at least 77 voxels. Subsequently, the size, cerebral region and MNI coordinates of the clusters with significantly different ReHo values were obtained.

The present study aimed to determine whether the ReHo values varied with disease progression among patients with strabismic amblyopia. Pearson's correlation analysis was used to determine the association between the ReHo indices of the identified regions and the best-corrected visual acuity.
Table I. Basic characteristics of strabismic amblyopia group and normal control group.

\begin{tabular}{lcc}
\hline Characteristic & $\mathrm{SA}(\mathrm{n}=12)$ & $\mathrm{NC}(\mathrm{n}=34)$ \\
\hline Gender (M/F) & $4 / 8$ & $12 / 22$ \\
Age $($ years $)$ & $23.92 \pm 5.31$ & $24.09 \pm 2.78$ \\
AE or NDE (L/R) & $5 / 7$ & $24 / 10$ \\
BCVA (logMAR) of & & \\
AE or NDE & $1.19 \pm 0.44$ & $0.00 \pm 0.00$
\end{tabular}

SA, strabismic amblyopia; NC, normal control; M, male; F, female; AE, amblyopic eye; NDE, nondominant eye; L, left; R, right; BCVA, best corrected visual acuity.

Furthermore, to evaluate the association between ReHo values and age, the same correlation analysis was performed. $\mathrm{P}<0.05$ was used to indicate a statistically significant difference. Finally, receiver operating characteristic (ROC) curve analysis was performed to evaluate the sensitivity and specificity of ReHo values from altered brain regions to distinguish subjects with strabismic amblyopia from normal subjects.

\section{Results}

Comparison of demographic parameters between normal controls and patients with amblyopia. As presented in Table I, the patients with amblyopia included four males and eight females aged $23.92 \pm 5.31$ years. A total of 5 patients were affected in the left eye and 7 patients were affected in the right eye. A total of 7 patients exhibited esotropic amblyopia and 5 patients exhibited exotropic amblyopia. The normal control group included 12 males and 22 females aged $24.09 \pm 2.78$ years. The gender distribution $\left(\chi^{2}=0.015 ; \mathrm{P}=1.00\right)$ and age $(\mathrm{t}=0.107 ; \mathrm{P}=0.917)$ were not significantly different between the two groups.

Differences in the ReHo values between the two groups. Significant differences in the ReHo values of the visual cortices were identified between patients and controls (Table II). Compared with those in the control group, patients with strabismic amblyopia exhibited significantly decreased ReHo values of certain parts of the occipital lobe, including the cuneus, lingual gyrus and superior occipital gyrus (Fig. 1). However, certain brain areas exhibited significantly increased ReHo values, including the precuneus and medial prefrontal cortex (Fig. 1).

Association between the ReHo values and clinical manifestations of patients with strabismic amblyopia. There was no correlation between the ReHo index and visual acuity of patients with strabismic amblyopia (Fig. 2). The ReHo index of the precuneus was negatively correlated with age $(r=-0.664$, $\mathrm{P}=0.019$ ) in the amblyopic group, but no such correlation was present in the occipital lobe and medial prefrontal cortex (Fig. 3). Furthermore, there was no correlation between the ReHo index and age in any of the above-mentioned regions in the healthy controls (Fig. 3). 
Table II. Differences of ReHo in brain functional activities between the strabismic amblyopia group and normal control group.

\begin{tabular}{lccc}
\hline Brain region & $\begin{array}{c}\text { Brodmann } \\
\text { region }\end{array}$ & $\begin{array}{c}\text { T-value } \\
\text { (voxels) }\end{array}$ & $\begin{array}{c}\text { Coordinates in MNI } \\
(\mathrm{x}, \mathrm{y}, \mathrm{z})\end{array}$ \\
\hline $\begin{array}{l}\text { Decreased ReHo in strabismic amblyopia } \\
\text { Certain parts of the occipital lobe }\end{array}$ & $17 / 18$ & -3.6055 & 128 \\
$\begin{array}{l}\text { (cuneus, lingual gyrus and } \\
\text { superior occipital gyrus) }\end{array}$ & & & $(21,-99,-9)$ \\
$\begin{array}{l}\text { Increased ReHo in strabismic amblyopia } \\
\text { Precuneus } \\
\text { Medial prefrontal cortex } \\
\text { (superior frontal gyrus and middle frontal gyrus) }\end{array}$ & 7 & 4.3857 & 104 \\
\end{tabular}

MNI, Montreal Neurological Institute; ReHo, regional homogeneity.
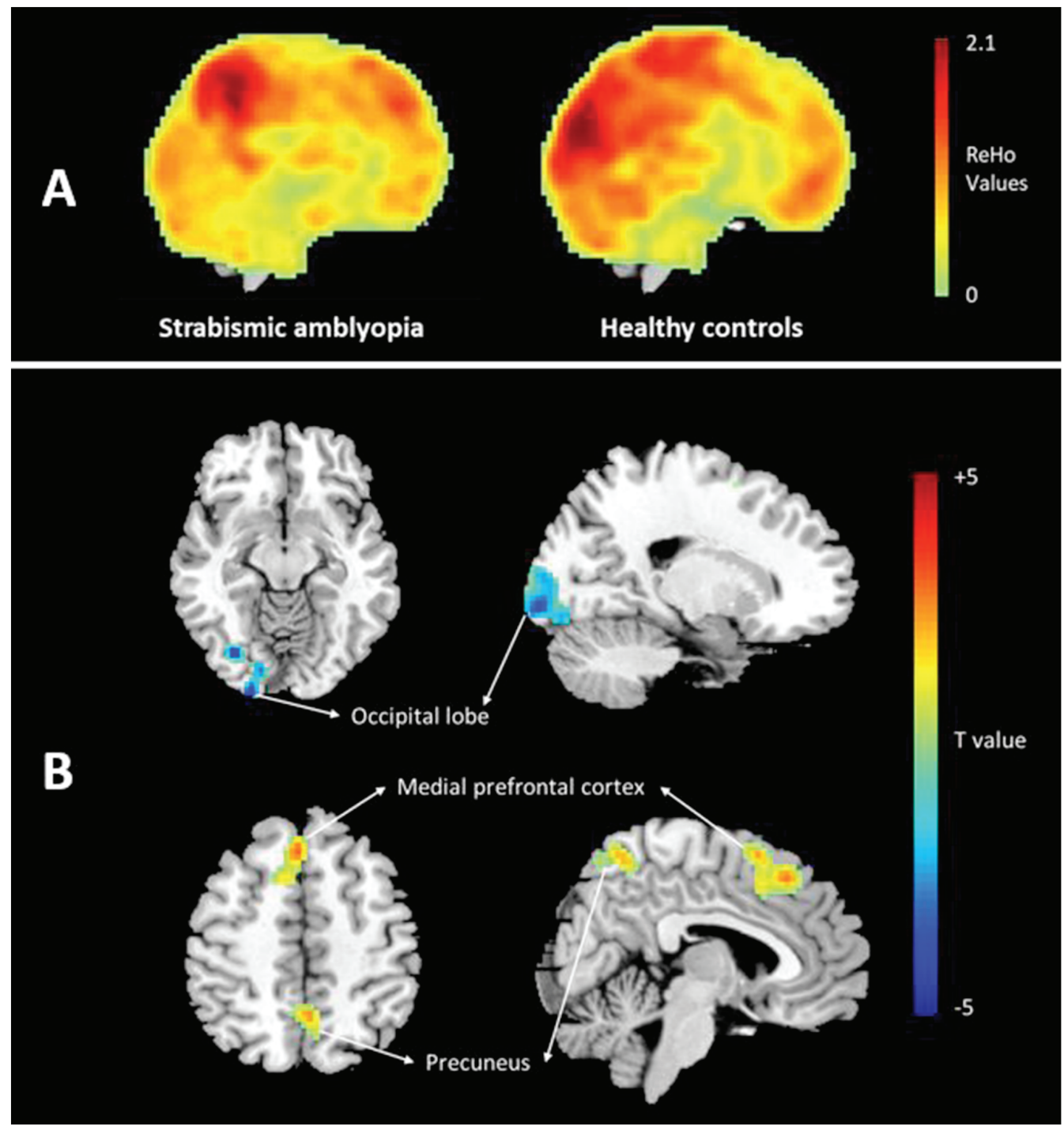

Figure 1. (A) Representative ReHo images of a patient and a control subject. (B) Cerebral regions with abnormal ReHo values in patients with strabismic amblyopia compared with the normal control group. The regions that are shown in red colour here are those that have elevated ReHo values compared with those in the control group and blue colour stands for reduced ReHo values. ReHo, regional homogeneity.

ROC curve analysis revealing ReHo in the medial prefrontal cortex is able to distinguish strabismic amblyopia from normal controls. As presented in Fig. 4, ROC curve analysis demonstrated that the greatest area under curve (AUC) value 

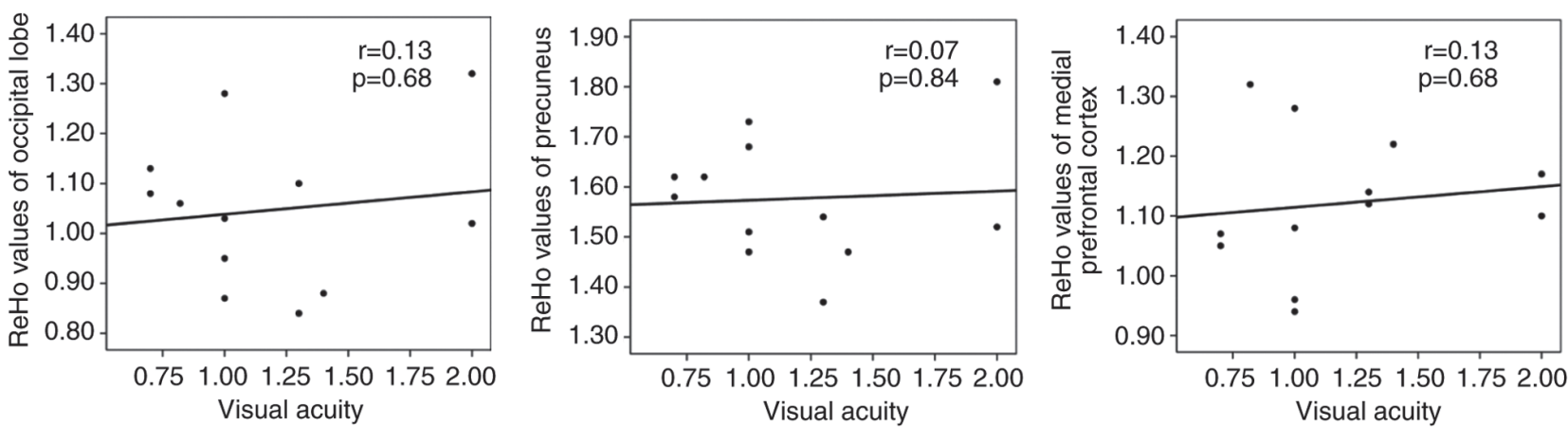

Figure 2. No correlation was determined between ReHo values of abnormal brain regions and visual acuity in subjects with strabismic amblyopia. ReHo, regional homogeneity.
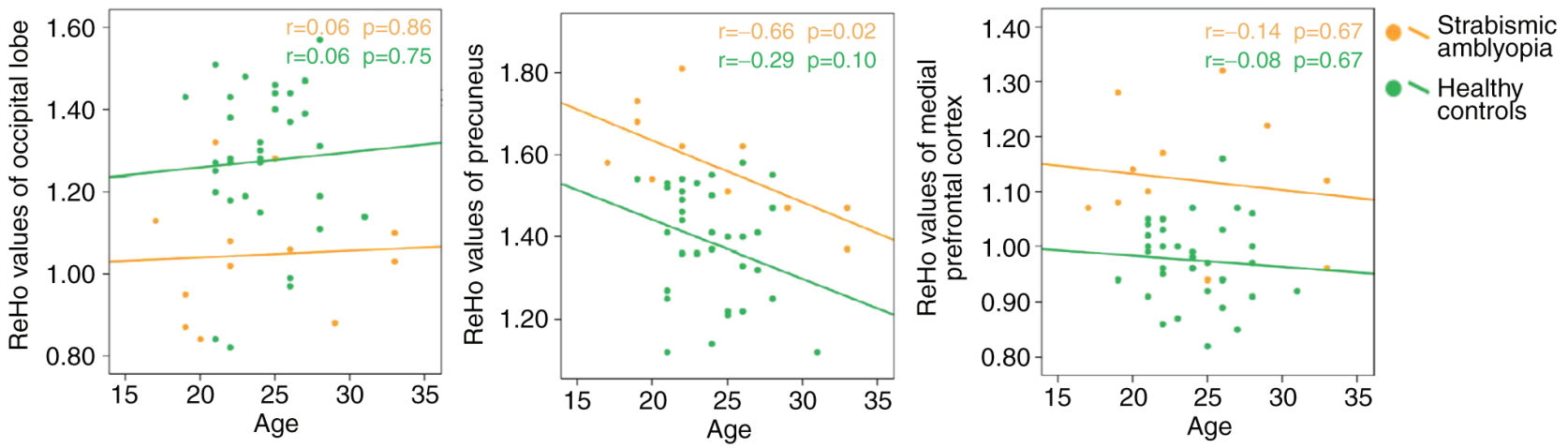

Figure 3. Correlation analysis between ReHo value of abnormal brain region and age in the two groups. The ReHo index of the precuneus was negatively correlated with age in the amblyopic group, but there was no such correlation in the occipital lobe and medial prefrontal cortex in the amblyopic group or in any of the above regions in the healthy controls. ReHo, regional homogeneity.
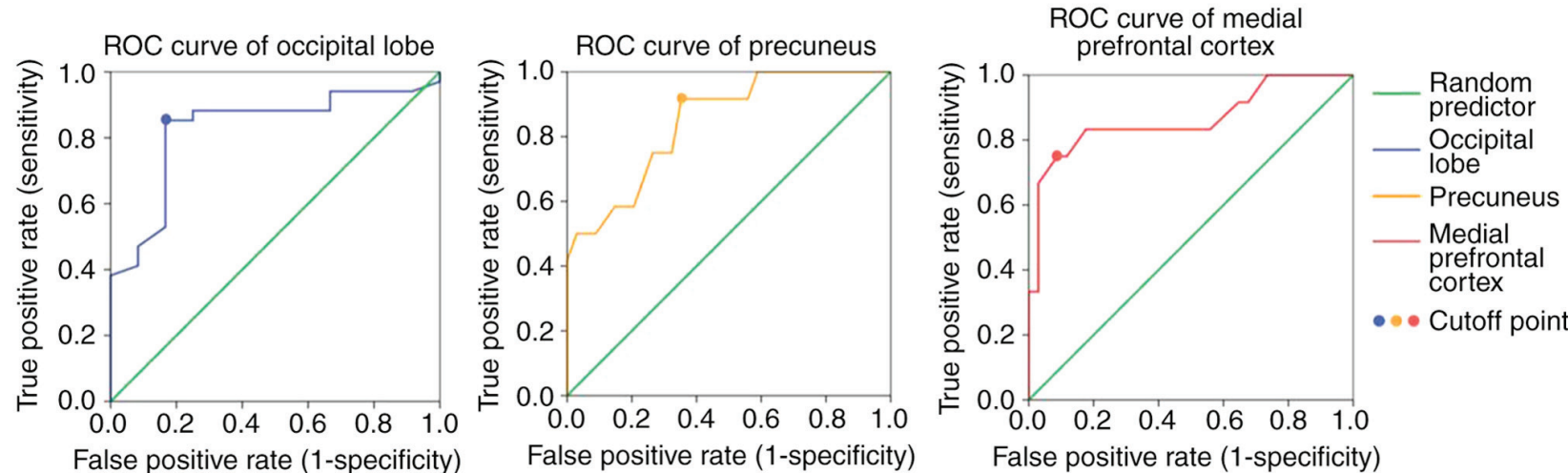

Figure 4. ROC curves for differentiating patients with strabismic amblyopia from normal controls based on the ReHo value in the precuneus, occipital lobe and medial prefrontal cortex. ROC, receiver operating characteristic.

was in the medial prefrontal cortex (AUC $=0.864)$, and the ReHo value was able to discriminate patients with strabismic amblyopia from normal controls with a sensitivity of $75 \%$ and specificity of $91.2 \%$. The AUC of precuneus and occipital lobe was 0.846 and 0.828 , respectively.

\section{Discussion}

To the best of our knowledge, the ReHo index was not previously used to investigate resting-state spontaneous brain activity in patients with strabismic amblyopia. Decreased spontaneous brain activity represented by reduced ReHo values was detected in certain parts of the occipital lobe, including the cuneus, lingual gyrus and superior occipital gyrus. However, increased ReHo values were determined in the precuneus and certain parts of the prefrontal cortex, including the superior frontal gyrus and middle frontal gyrus of patients with strabismic amblyopia compared with normal controls.

Mendola et al (2) proposed that amblyopia may be associated with structural brain abnormalities. This study used voxel-based analysis of MRI and detected decreased visual 
cortex gray-matter volume among children and adult patients with amblyopia (2). A previous study by our group used magnetic resonance diffusion tensor imaging and diffusion tensor tractography to identify decreased compactability, integrity and directivity of optic radiations among patients with anisometropic amblyopia (28). Furthermore, previous studies identified decreased white-matter volume among patients with amblyopia $(3,4)$. These structural abnormalities may contribute to alterations of spontaneous brain activity detected in the present study using resting-state fMRI.

In the present study, patients with strabismic amblyopia exhibited significantly decreased ReHo values in certain parts of the occipital lobe, including the cuneus, lingual gyrus and superior occipital gyrus, which belong to the Brodmann area (BA) 17 and 18. BA 17, also known as the striate cortex, V1 area or primary visual cortex, is used for visual information processing. BA 18, also known as the $\mathrm{V} 2$ area, is a part of the extrastriate cortex used for further processing of visual information transformed from the striate cortex. The present results are consistent with those of numerous previous studies. Wiesel and Hubel (29) proposed that form deprivation including occlusion, congenital cataract and ptosis may affect the visual cortex and identified amblyopia-associated alterations in the V1 area. Subsequently, numerous studies demonstrated that abnormal visual experiences may lead to morphological and functional alterations of the V1 area (30-35). A previous study by our group used task-based fMRI to further demonstrate that the activation of the V1 and V2 areas was reduced among patients with amblyopia (10). These results suggested that the visual information processing of the brain is deteriorated in strabismic amblyopia.

However, using the same ReHo analysis method based on resting-state fMRI as that described in the present study, Lin et al (12) did not identify any alterations in the ReHo index of the V1 area among patients with anisometropic amblyopia. Furthermore, increased ReHo values were identified in the V2 and V3 areas (12). The above results may reflect the differences in the neural deficit between patients with anisometropic and strabismic amblyopia.

In the present study, patients with strabismic amblyopia exhibited increased ReHo values in the precuneus and medial prefrontal cortex, including the superior frontal gyrus and middle frontal gyrus, compared with those in normal controls. The precuneus is located in the medial wall of BA 7, a component of the dorsal visual pathway receiving visual information from the middle temporal area, also known as area V5. The major functions of the dorsal stream, also known as the 'where pathway' or the 'how pathway', include motion detection, object localization and arm-eye coordination (13). The precuneus participates in a number of highly integrated tasks, including visuomotor coordination $(36,37)$, visuospatial imagery, episodic memory retrieval and self-processing operations, including taking of the first-person perspective and the experience of agency (38). Niechwiej-Szwedo et al $(39,40)$ identified abnormal visuomotor processes in patients with strabismic amblyopia using behavioral experiments. Contrary to the results of those behavioral experiments, the increased ReHo values detected in the present study in the precuneus of patients with strabismic amblyopia may reflect the plasticity of the human brain compensating for visuomotor coordination and visuospatial imagery deficits.

The medial prefrontal cortex is located in BA 10, an essential component of the default-mode network associated with consciousness (41). Of note, strabismic amblyopes frequently suffer from high anxiety (42). First, their appearance is changed due to deviation. Furthermore, strabismic amblyopes have an increased risk of blindness when the fellow eye is injured or diseased, due to the low visual acuity of the amblyopic eye, leading to patients becoming more anxious. This may be associated with the increased ReHo values in the medial prefrontal cortex, and may also reflect compensatory plasticity.

The potential compensatory mechanism has also been reported in patients with visual impairments, including blindness $(43,44)$, optic neuritis $(45)$ and amblyopia, as indicated by the results of task-based fMRI (46).

In the present study, no correlation between the standardized ReHo values and the visual acuity of patients with amblyopia was determined. Furthermore, a number of previous task-based fMRI studies revealed no association between visual acuity and the activation of cerebral regions under visual stimulation among patients with amblyopia $(47,48)$. In addition, a resting-state fMRI study by Liang et al (16) revealed no correlation between the ALFF values and the visual acuity of patients with amblyopia. These results suggested that the visual impairment of patients with amblyopia may not be associated with functional deficits in the cortex. Among adult patients with amblyopia, visual acuity alone did not accurately reflect cortical defects. In the present study, the ReHo index of the precuneus was negatively correlated with age, which suggested that the plasticity of the precuneus decreases with age.

ROC curve analysis revealed that the ReHo value of the medial prefrontal cortex is the best value to distinguish patients with strabismic amblyopia from normal subjects.

Of note, the present study had several limitations. The number of patients included in the analysis was small due to difficulties in the recruitment of individuals with adult strabismic amblyopia. Thus, no multi-parameter analysis was performed and the results should be considered preliminary. In addition, the present study did not differentiate between right- and left-eye defects due to the small sample size, and the results should be interpreted with caution. Furthermore, patient age may influence brain plasticity and the ReHo characteristics of pediatric patients with amblyopia remain elusive; however, the present study included age-matched patient and control groups. Finally, the enrolled subjects were all relatively young and the correlation between ReHo values and age was only assessed in a relatively narrow age range. Future studies on amblyopia should include spontaneous brain activity data from a large cohort of pediatric and adult patients, and add further parameters, including the duration of strabismic amblyopia in patients, into the correlation analysis.

In conclusion, in the present study, resting-state fMRI and ReHo analysis were used to detect visual information processing impairment in the V1 and V2 areas in adult anisometropic amblyopia. The ReHo index of the precuneus was negatively correlated with age. However, no correlation 
was observed between ReHo values and patient visual acuity. ROC curve analysis demonstrated that the ReHo in the medial prefrontal cortex was able to distinguish strabismic amblyopia from normal controls. The results suggested that among adult patients with strabismic amblyopia, brain plasticity compensated for visuomotor coordination and visuospatial imagery deficits.

\section{Acknowledgements}

Not applicable.

\section{Funding}

The present study was supported by the Sichuan Science and Technology Program (grant no. 2018SZ0146).

\section{Availability of data and materials}

The datasets used and/or analyzed during the current study are available from the corresponding author on reasonable request.

\section{Authors' contributions}

XY and LL designed the method. XY drafted the manuscript. QL collected patient data. QL and LL analyzed the data. LL, QG and XH conceptualized the topic of the current study and interpreted the data. LL revised the manuscript. All authors read and approved the final manuscript.

\section{Ethics approval and consent to participate}

The experimental protocol was approved by the institutional review board of West China Hospital of Sichuan University (Chengdu, China; approval no. 201433). All subjects provided written informed consent to participate in the study.

\section{Patient consent for publication}

The participants provided written informed consent for the publication of their MRI images.

\section{Competing interests}

The authors declare that they have no competing interests.

\section{References}

1. Xiao JX, Xie S, Ye JT, Liu HH, Gan XL, Gong GL and Jiang XX: Detection of abnormal visual cortex in children with amblyopia by voxel-based morphometry. Am J Ophthalmol 143: 489-493, 2007.

2. Mendola JD, Conner IP, Roy A, Chan ST, Schwartz TL, Odom JV and Kwong KK: Voxel-based analysis of MRI detects abnormal visual cortex in children and adults with amblyopia. Hum Brain Mapp 25: 222-236, 2005.

3. Li Q, Jiang Q, Guo M, Li Q, Cai C and Yin X: Grey and white matter changes in children with monocular amblyopia: Voxel-based morphometry and diffusion tensor imaging study. Br J Ophthalmol 97: 524-529, 2013.

4. Yan X, Lin X, Wang Q, Zhang Y, Chen Y, Song S and Jiang T: Dorsal visual pathway changes in patients with comitant extropia. PLoS One 5: e10931, 2010.
5. Goodyear BG, Nicolle DA, Humphrey GK and Menon RS: BOLD fMRI response of early visual areas to perceived contrast in human amblyopia. J Neurophysiol 84: 1907-1913, 2000.

6. Barnes GR, Hess RF, Dumoulin SO, Achtman RL and Pike GB: The cortical deficit in humans with strabismic amblyopia. J Physiol 533: 281-297, 2001.

7. Hess RF, Thompson B, Gole G and Mullen KT: Deficient responses from the lateral geniculate nucleus in humans with amblyopia. Eur J Neurosci 29: 1064-1070, 2009.

8. Miki A, Liu GT, Goldsmith ZG, Liu CS and Haselgrove JC: Decreased activation of the lateral geniculate nucleus in a patient with anisometropic amblyopia demonstrated by functional magnetic resonance imaging. Ophthalmologica 217: 365-369, 2003.

9. Ho CS and Giaschi DE: Low- and high-level motion perception deficits in anisometropic and strabismic amblyopia: Evidence from fMRI. Vision Res 49: 2891-2901, 2009.

10. Li H, Yang X, Gong Q, Chen H, Liao M and Liu L: BOLD responses to different temporospatial frequency stimuli in V1 and V2 visual cortex of anisometropic amblyopia. Eur J Ophthalmol 23: 147-155, 2013.

11. Raichle ME and Mintun MA: Brain work and brain imaging. Annu Rev Neurosci 29: 449-476, 2006.

12. Lin X, Ding K, Liu Y, Yan X, Song S and Jiang T: Altered spontaneous activity in anisometropic amblyopia subjects: Revealed by resting-state FMRI. PLoS One 7: e43373, 2012.

13. Ding K, Liu Y, Yan X, Lin X and Jiang T: Altered functional connectivity of the primary visual cortex in subjects with amblyopia. Neural Plast 2013: 612086, 2013.

14. Wang T, Li Q, Guo M, Peng Y, Li Q, Qin W and Yu C: Abnormal functional connectivity density in children with anisometropic amblyopia at resting-state. Brain Res 1563: 41-51, 2014.

15. Tang A, Chen T, Zhang J, Gong Q and Liu L: Abnormal spontaneous brain activity in patients with anisometropic amblyopia using resting-state functional magnetic resonance imaging. J Pediatr Ophthalmol Strabismus 54: 303-310, 2017.

16. Liang M, Xie B, Yang H, Yu L, Yin X, Wei L and Wang J: Distinct patterns of spontaneous brain activity between children and adults with anisometropic amblyopia: A resting-state fMRI study. Graefes Arch Clin Exp Ophthalmol 254: 569-576, 2016.

17. Liang M, Xie B, Yang H, Yin X, Wang H, Yu L, He S and Wang J: Altered interhemispheric functional connectivity in patients with anisometropic and strabismic amblyopia: A resting-state fMRI study. Neuroradiology 59: 517-524, 2017.

18. Zang Y, Jiang T, Lu Y, He Y and Tian L: Regional homogeneity approach to fMRI data analysis. Neuroimage 22: 394-400, 2004.

19. Liao H, Wang L, Zhou B, Tang J, Tan L, Zhu X, Yi J, Chen X and Tan C: A resting-state functional magnetic resonance imaging study on the first-degree relatives of persons with schizophrenia. Brain Imaging Behav 6: 397-403, 2012.

20. Yoo JH, Oh Y, Jang B, Song J, Kim J, Kim S, Lee J, Shin HY, Kwon JY, Kim YH, et al: The effects of equine-assisted activities and therapy on resting-state brain function in attention-deficit/hyperactivity disorder: A pilot study. Clin Psychopharmacol Neurosci 14: 357-364, 2016.

21. Wu T, Long X, Zang Y, Wang L, Hallett M, Li K and Chan P: Regional homogeneity changes in patients with Parkinson's disease. Hum Brain Mapp 30: 1502-1510, 2009.

22. Sorg C, Riedl V, Perneczky R, Kurz A and Wohlschläger AM: Impact of Alzheimer's disease on the functional connectivity of spontaneous brain activity. Curr Alzheimer Res 6: 541-553, 2009.

23. Liu C, Liu Y,Li W, Wang D, Jiang T, Zhang Y and Yu C: Increased regional homogeneity of blood oxygen level-dependent signals in occipital cortex of early blind individuals. Neuroreport 22 : 190-194, 2011.

24. Song Y, Mu K, Wang J, Lin F, Chen Z, Yan X, Hao Y, Zhu W and Zhang H: Altered spontaneous brain activity in primary open angle glaucoma: A resting-state functional magnetic resonance imaging study. Plos One 9: e89493, 2014.

25. Huang X, Li SH, Zhou FQ, Zhang Y, Zhong YL, Cai FQ, Shao Y and Zeng XJ: Altered intrinsic regional brain spontaneous activity in patients with comitant strabismus: A resting-state functional MRI study. Neuropsychiatr Dis Treat 12: 1303-1308, 2016.

26. Society CO: Expert consensus on amblyopia diagnosis. Chin J Ophthalmol 47: 768, 2011

27. Hou Y, Yang J, Luo C, Ou R, Song W, Liu W, Gong Q and Shang H: Patterns of striatal functional connectivity differ in early and late onset Parkinson's disease. J Neurol 263: 1993-2003, 2016. 
28. Song HY, Qi S, Tang HH, Yu FJ and Liu LQ: MR DTI and DTT study on the development of optic radiation in patients with anisometropia amblyopia. Sichuan Da Xue Xue Bao Yi Xue Ban 41: 648-651, 2010 (In Chinese).

29. Wiesel TN and Hubel DH: Single-cell responses in striate cortex of kittens deprived of vision in one Eye. J Neurophysiol 26: 1003-1017, 1963.

30. Tychsen L, Wong AM and Burkhalter A: Paucity of horizontal connections for binocular vision in V1 of naturally-strabismic macaques: Cytochrome- oxidase compartment specificity. J Comp Neurol 474: 261-275, 2004.

31. Kiorpes L: Visual processing in amblyopia: Animal studies Strabismus 14: 3-10,2006.

32. Mitchell DE, Sengpiel F, Hamilton DC, Schwarzkopf DS and Kennie J: Protection against deprivation amblyopia depends on relative not absolute daily binocular exposure. J Vis 11: 13, 2011

33. Mitchell DE, Kennie J, Schwarzkopf DS and Sengpiel F: Daily mixed visual experience that prevents amblyopia in cats does not always allow the development of good binocular depth perception. J Vis 9: 22.1-7, 2009.

34. Sengpiel F: Experimental models of amblyopia: Insights for prevention and treatment. Strabismus 19: 87-90, 2011.

35. Norcia AM, Hale J, Pettet MW, McKee SP and Harrad RA Disparity tuning of binocular facilitation and suppression after normal versus abnormal visual development. Invest Ophthalmol Vis Sci 50: 1168-1175, 2009.

36. Hadjidimitrakis K, Breveglieri R, Placenti G, Bosco A, Sabatini SP and Fattori P: Fix your eyes in the space you could reach: Neurons in the macaque medial parietal cortex prefer gaze positions in peripersonal space. PLoS One 6: e23335, 2011.

37. Milner AD and Goodale MA: Two visual systems re-viewed. Neuropsychologia 46: 774-785, 2008.

38. Cavanna AE and Trimble MR: The precuneus: A review of its functional anatomy and behavioural correlates. Brain 129: 564-583, 2006

39. Niechwiej-Szwedo E, Goltz HC, Chandrakumar M and Wong AM: Effects of strabismic amblyopia on visuomotor behavior: Part II. Visually guided reaching. Invest Ophthalmol Vis Sci 55: 3857-3865, 2014.
40. Niechwiej-Szwedo E, Goltz HC, Chandrakumar M and Wong AM: Effects of strabismic amblyopia and strabismus without amblyopia on visuomotor behavior: III. Temporal eye-hand coordination during reaching. Invest Ophthalmol Vis Sci 55: 7831-7838, 2014

41. Frith C: The role of the prefrontal cortex in self-consciousness: The case of auditoryhallucinations. Philos Trans R Soc Lond B Biol Sci 351: 1505-1512, 1996.

42. Sim B, Yap GH and Chia A: Functional and psychosocial impact of strabismus on Singaporean children. J AAPOS 18: 178-182, 2014.

43. Yu C, Liu Y, Li J, Zhou Y, Wang K, Tian L, Qin W, Jiang T and $\mathrm{Li}$ K: Altered functional connectivity of primary visual cortex in early blindness. Hum Brain Mapp 29: 533-543, 2008.

44. Yu C, Shu N, Li J, Qin W, Jiang T and Li K: Plasticity of the corticospinal tract in early blindness revealed by quantitative analysis of fractional anisotropy based on diffusion tensor tractography. Neuroimage 36: 411-417, 2007.

45. Liang P, Liu Y, Jia X, Duan Y, Yu C, Qin W, Dong H, Ye J and Li K: Regional homogeneity changes in patients with neuromyelitis optica revealed by resting-state functional MRI. Clin Neurophysiol 122: 121-127, 2011.

46. Conner IP, Odom JV, Schwartz TL and Mendola JD: Retinotopic maps and foveal suppression in the visual cortex of amblyopic adults. J Physiol 583: 159-173, 2007.

47. Li C, Cheng L, Yu Q, Xie B and Wang J: Relationship of visual cortex function and visual acuity in anisometropic amblyopic children. Int J Med Sci 9: 115-120, 2012.

48. Leguire LE, Algaze A, Kashou NH, Lewis J, Rogers GL and Roberts C: Relationship among fMRI, contrast sensitivity and visual acuity. Brain Res 1367: 162-169, 2011.

(i) (2) This work is licensed under a Creative Commons Attribution-NonCommercial-NoDerivatives 4.0 International (CC BY-NC-ND 4.0) License. 